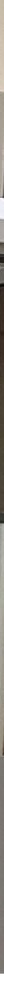

Internist 2021 - 62 (Suppl 5):S497-S498

https://doi.org/10.1007/s00108-021-01187-7 Angenommen: 23. September 2021 Online publiziert: 27. Oktober 2021

(c) Springer Medizin Verlag GmbH, ein Teil von Springer Nature 2021
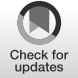

\title{
Facharzt-Training Innere Medizin - Das vierte Sonderheft ist da!
}

\author{
Anahita Fathi' · Moritz Hundertmark ${ }^{2}$. Matthias Raspe ${ }^{3}$ \\ '1. Medizinische Klinik und Poliklinik - Sektion Infektiologie, Universitätsklinikum Hamburg-Eppendorf, \\ Hamburg, Deutschland \\ ${ }^{2}$ Oxford Centre for Clinical Magnetic Resonance Research (OCMR), Division of Cardiovascular Medicine, \\ Radcliffe Department of Medicine, University of Oxford, Oxford, Großbritannien \\ ${ }^{3}$ Klinik m. S. Infektiologie und Pneumologie, Campus Virchow-Klinikum, Charité - Universitätsmedizin \\ Berlin, Berlin, Deutschland
}

Die ärztliche Weiterbildung ist für alle jungen Ärzt*innen in den ersten Berufsjahren ein zentrales Thema im Berufsalltag. Trotz Vorgaben der Weiterbildungsordnungen und Informationen der jeweiligen Ärztekammern bleibt die individuelle Weiterbildung für viele angehende Internist*innen eine Herausforderung. Gerade in der internistischen Weiterbildung müssen viele hochgradig komplexe Spezialisierungen integriert und die entsprechenden Kompetenzen für die Patientenversorgung erlernt werden. Bedauerlicherweise ist die Zeit, um diese Fähigkeiten zu entwickeln und therapeutische Entscheidungen zu reflek- tieren, unter dem Einfluss politisch geprägter Reformen und eines stetig wachsenden ökonomischen Fokus im Gesundheitswesen knapp geworden. Die Arbeitsverdichtung hat, nicht zuletzt durch den immer höheren Dokumentationsaufwand, spürbar zugenommen, während Vorgesetzten immer weniger Zeit für die Supervision und das Weitergeben von Wissen und Kompetenzen bleibt. Eine Kompensation für Kliniken, die sich einer besonderen Qualität in der Weiterbildung verschreiben und exzellente Weiterbildungsbedingungen bieten, existiert bislang nicht. Das Ergebnis ist eine deutliche Diskrepanz der Weiter- 
bildungsqualität zwischen Häusern, denen es ökonomisch gut genug geht, um sich Weiterbildung „leisten zu können" und hier einen Schwerpunkt zu setzen, und anderen, in denen überwiegend autodidaktisches "Learning by doing" betrieben wird.

Es bleibt zu hoffen, dass die sukzessive Implementierung der neuen Weiterbildungsordnung durch mehr und mehr Landesärztekammern eine spürbare Verbesserung bringen wird - auch wenn dies keinen wesentlichen Einfluss auf die Rahmenbedingungen im Gesundheitswesen haben kann. In der heutigen medizinischen Realität von Fallpauschalen und besserer Vergütung diagnostischer und therapeutisch-interventioneller Maßnahmen hat jede Klinik ein hohes Interesse, Berufsanfänger*innen in Bezug auf praktische Fähigkeiten möglichst schnell autark werden zu lassen. Wie steht es aber um die Entwicklung des theoretischen Wissens und diagnostischen Denkens, das integraler Bestandteil der Inneren Medizin ist?

Genau hier sehen wir den festen Platz für die DGIM e.Akademie der Deutschen

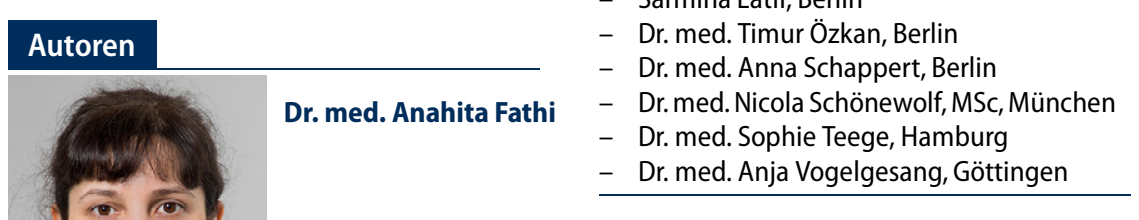

Gesellschaft für Innere Medizin. Dank ihr können Ärzt*innen in Weiterbildung frühzeitig und weiterbildungsbegleitend ihr theoretisches Wissen und diagnostisches Gespür überprüfen und ausbauen. Das Facharzt-Training Innere Medizin, das hier als viertes Sonderheft in der Printversion vorliegt, ist nicht nur in Print, sondern auch digital in der DGIM e.Akademie als E-Learning verfügbar. Es umfasst derzeit 100 Fälle aus allen Bereichen der Inneren Medizin mit Prüfungsfragen und Antworten. Diese sind nach neuestem wissenschaftlichem Stand und in ansprechendem digitalem Design umgesetzt. So wird ein effektives und kontinuierliches Lernen ermöglicht. Das Facharzt-Training Innere Medizin eignet sich ideal für die Vorbereitung auf die Facharztprüfung und bietet - auch zum Nachschlagen - einen guten Überblick über Erkrankungen, Diagnostik und Therapie. Das FacharztTraining Innere Medizin wird vom Wis-

\section{Infobox 2}

AG Junge DGIM

Die AG Junge DGIM setzt sich als Vertretung des internistischen Nachwuchses der Deutschen Gesellschaft für Innere Medizin (DGIM) für die Belange junger Internist*innen und den barrierefreien Zugang zu qualitativer Weiterbildung ein. Die AG umfasst mehr als 400 Mitglieder. Im sog. Arbeitskreis der AG sind mehr als 40 Mitglieder aktiv und organisieren u.a. jährlich im Rahmen des DGIM-Kongresses ein umfangreiches Nachwuchsprogramm. Interessierte sind immer willkommen und melden sich gerne unter:jungedgim@dgim.de

senschaftlichen Beirat der DGIM mit den Vorsitzenden bzw. federführenden Herausgebern Herrn Prof. Ertl und Herrn Prof. Arlt herausgegeben (DInfobox 1).

Nutzen Sie die DGIM e.Akademie, sie ist überall verfügbar!

Wir wünschen Ihnen viel Freude mit diesem vierten Heft und viel Erfolg auf Ihrem Weg zum internistischen Facharzt!

Für die AG Junge DGIM (•Infobox 2) Dr. med. Anahita Fathi (Sprecherin), Dr. med. Moritz Hundertmark (Sprecher) und

Dr. med. Matthias Raspe (ehemaliger Sprecher)

\section{Korrespondenzadresse}

\section{Dr. med. Anahita Fathi}

1. Medizinische Klinik und Poliklinik - Sektion Infektiologie, Universitätsklinikum HamburgEppendorf

Martinistr. 52, 20246 Hamburg, Deutschland a.fathi@uke.de

\section{Dr. med. Moritz Hundertmark}

Oxford Centre for Clinical Magnetic Resonance Research (OCMR), Division of Cardiovascular Medicine, Radcliffe Department of Medicine, University of Oxford Oxford, Großbritannien moritz.hundertmark@stcatz.ox.ac.uk

\section{Dr. med. Matthias Raspe}

Klinik m. S. Infektiologie und Pneumologie, Campus Virchow-Klinikum, Charité Universitätsmedizin Berlin

Augustenburger Platz 1, 13353 Berlin, Deutschland

matthias.raspe@charite.de

Interessenkonflikt. A. Fathi, M. Hundertmark und M. Raspe geben an, dass kein Interessenkonflikt besteht. 